\title{
Grupos eTwinning - A Percepção dos Professores sobre uma Comunidade de Prática
}

\section{eTwinning Groups - Teachers' Perception on a Community of Practice}

\author{
Rita Zurrapa*, João Marques** \\ *Agrupamento de Escolas Agualva Mira Sintra; **Agrupamento de Escolas de Figueiró do Vinhos
}

\begin{abstract}
Resumo
O projecto eTwinning apresenta-se como a Comunidade de Escolas da Europa. O eTwinning promove a colaboração dos professores com a utilização das ferramentas digitais. O presente artigo debruça-se sobre uma das ferramentas disponíveis na plataforma eTwinning que promove a aprendizagem entre pares, os Grupos eTwinning. Estes são espaços privados dentro da plataforma onde os professores se reúnem para discutir um tema específico, com o objectivo de partilhar vivências, metodologias, saberes. $\mathrm{O}$ presente artigo debruça-se sobre uma das ferramentas disponíveis que promovem a aprendizagem entre pares, os Grupos eTwinning.

Palavras chave: eTwinning; comunidades de prática; redes sociais
\end{abstract}

\begin{abstract}
The eTwinning project is the Community of School in Europe. eTwinning promotes the collaboration among teachers using digital tools. This article focuses on one of the tools that can be found in the eTwinning platform that promotes peer learning, the eTwinning Groups. These are private spaces in the platform where teachers gather to discuss one specific theme, with the aim of sharing experiences, methodologies, and knowledge. This article intends to focus on what teachers look for in these learning spaces.
\end{abstract}

\section{Introdução}

O eTwinning é a maior comunidade de escolas da Europa. É um projecto da Comissão Europeia que promove a colaboração dos professores e alunos através do uso das tecnologias.

O projecto eTwinning foi lançado em Janeiro de 2005 pelo Comissário Europeu Jan Figel numa Conferência em Bruxelas que reuniu 300 professores. Na altura tinha como objectivo identificar escolas que desejassem trabalhar em conjunto para dar oportunidade aos alunos de aprenderem e usarem as competências digitais, assim como, promover a consciência da multiculturalidade da Europa. A Comissão Europeia propôs que o eTwinning fosse o catalisador para intensificar a cooperação já existente nas escolas. (Crawley, Gilleran, Scimeca, Vuorikari, Wastiau. 2009)

No primeiro ano o eTwinning reuniu 12000 escolas e 12200 professores dos 28 países que pertenciam na altura ao projecto. No primeiro ano surgiram 1000 parcerias. À data deste artigo o projecto tem 468265 professores envolvidos, oriundos de 179005 escolas de 42 países parceiros.

O projecto eTwinning foi lançado como a primeira acção do Programa eLearning da Comissão Europeia. Em 2008, inicia-se a segunda fase da sua existência integrado no Programa de Aprendizagem ao Longo da Vida (Lifelong Learning Programme (LLP), "eTwinning (as part of the Comenius action) started playing an essential role in education, far beyond what was initially foreseen" (Crawley, Gilleran, Scimeca, Vuorikari, Wastiau. 2009. p.3). Em 2014, entra na terceira fase passando a integrar o Programa Erasmus+.

Desde 2005 e com o passar dos anos a plataforma sofreu várias alterações tornando-se um espaço promotor de colaboração e de constante aprendizagem entre pares.

\section{O Portal eTwinning - espaço de colaboração}

O Portal do eTwinning é composto por diferentes áreas que podem ser acedidas a partir da página www.etwinning.net. $\mathrm{O}$ usuário que não estiver registado tem apenas acesso à primeira área onde pode encontrar toda a informação sobre as potencialidades de pertencer a esta comunidade, visitar uma galeria com alguns projectos destacados e aceder às publicações da responsabilidade do Serviço Central de Apoio (Central Support Service - CSS). Apenas os professores podem registar-se para entrar no espaço restrito que dá acesso a outras duas áreas do eTwinning. É nestas áreas que os professores interagem e aprendem uns com os outros.

O projecto eTwinning começou por ser um espaço que promovia a partilha entre os professores mas depressa houve necessidade de crescer e acompanhar os desejos dos seus usuários de fomentar a aprendizagem tornando-se uma comunidade onde a interacção e a colaboração entre os seus membros se tornou constante.

$\mathrm{O}$ projecto eTwinning surge numa altura em que a Internet era acessível a muitos professores que já a utilizavam com fins educativos. $\mathrm{O}$ eTwinning traz-lhes a oportunidade de encontrar parceiros com os mesmos interesses para realizarem trabalho colaborativo, ter apoio, ter oportunidades de formação e reconhecimento do trabalho realizado por eles e pelos seus alunos. 
Quando é lançada a plataforma teve como objectivo principal oferecer um espaço onde os professores pudessem encontrar parceiros e formar parcerias. Nos primeiros 3 anos de existência percebeu-se que os professores começaram a usar a plataforma para comunicar e aprender uns com os outros fora ou em paralelo com os projectos que realizavam. "In a way, eTwinning filled the need of a place, at international level, where teachers could find each other for a project involving their classes, but also a safe environment to grow together as professionals" (Crawley, Gerhard, Gilleran, Joyce, 2010, p.12)

Em 2007, a plataforma tinha cerca de 38000 professores registados e era um espaço que continuava a crescer, "eTwinning has slowly, but firmly, become "the place to join' for all teachers interested in going beyond their school's horizon, meeting colleagues in Europe, and helping their pupils work together with foreign peers." (Crawley, Gilleran, Scimeca, Vuorikari, Wastiau, 2009, p4)

Em 2008, é lançada a nova plataforma que procura aproximar a realidade do projecto à tendência da Web 2.0 incentivando mais a colaboração entre os professores, que passou a ter mais ferramentas que incentivavam a comunicação e a colaboração. "Significantly, the eTwinning motto has changed from School partnerships in Europe to The community for schools in Europe." (Crawley, Gilleran, Scimeca, Vuorikari, Wastiau, 2009, p.6).

\section{Grupos eTwinning - Espaço de desenvolvimento pessoal e profissional}

O espaço designado por eTwinning Live surge em 2015 transformando a forma dos professores interagirem entre si na plataforma. O eTwinning Live manteve algumas das ferramentas da plataforma anterior, contudo introduz outras novas que criam uma dinâmica semelhante às ferramentas de redes sociais mais populares promovendo a interacção e a aprendizagem entre pares.

A ferramenta do eTwinning Live que focamos neste artigo é "Grupos". Os projectos continuam a ser o que atrai muitos professores à comunidade mas a necessidade premente dos professores de aprender mais, tornou o eTwinning num espaço de formação tendo a ferramenta dos Grupos contribuído para isso. Os professores eTwinners perceberam que a colaboração e aprendizagem entre pares promove a mudança. Os professores aderiram de imediato à ferramenta, participando activamente logo após o seu lançamento.

A ferramenta Grupos promove dois géneros de espaços onde os professores podem aprender e partilhar conhecimentos, a saber Grupos de Destaque e Grupos criados pelos professores para professores.

Os Grupos eTwinning nascem no final de 2008 com o lançamento de um projecto piloto com três grupos temáticos, um para professores de matemática, ciência e tecnologia (MST), um para professores directores de escola e outro para professores interessados no tema da criatividade na educação. Este último grupo mantém-se activo, tendo por designação Creative Classroom e é o Grupo eTwinning com mais membros registados. Os restantes acabaram por renascer com novo formato e novos moderadores passado algum tempo. Nos anos seguintes surgiram outros Grupos, sendo que os que tiveram mais destaque foram o que reuniam os Embaixadores eTwinning e professores que partilhavam experiências dentro da temática "Using Media”. (Crawley, Gerhard, Gilleran, Joyce, 2010)

Tal como em 2010, actualmente os membros dos Grupos eTwinning participam nestes para aprender e partilhar.

O seu grande desenvolvimento só se dá com a última actualização da plataforma em 2015. Os Grupos eTwinning passam a ter características mais interactivas e dão a oportunidade a qualquer eTwinner ser um perito. Actualmente existem dois formatos de Grupos eTwinning: Grupos de Destaque (Featured Groups) (grupos da responsabilidade do Serviço Central de Apoio (CSS)) e grupos da responsabilidade do professor que os cria.

Entre as várias ferramentas que compõem a plataforma eTwinning encontramos no espaço privado, eTwinning Live, no quarto separador, a ferramenta Grupos, onde os professores podem criar novos grupos, entrar nos seus próprios grupos, nos grupos dos quais se tornaram membros, sejam estes de destaque ou de outros professores e podem pesquisar outros grupos através do motor de busca da plataforma.

Qualquer eTwinner pode criar um Grupo eTwinning. Para tal basta seleccionar "Criar um grupo" e preencher o questionário. Para evitar a repetição de grupos temáticos semelhantes os eTwinners são aconselhados a fazer uma pesquisa antes de registarem um novo grupo. O grupo é aprovado pelo Serviço Central de Apoio. Para tal é necessário que o professor que pretende criar o grupo que escreva as metas e objectivos do grupo em Inglês, não obstante o grupo pode ser criado, organizado e desenvolvido em qualquer língua.

Os criadores dos grupos decidem se o grupo fica aberto a todos ou se é privado. Depois de aprovado o professor responsável pode convidar eTwinners através do eTwinning Live e pessoas que não sejam eTwinners, desta feita criando uma conta de visitante (estes últimos apenas terão acesso a este espaço e terão menos permissões que os eTwinners, permitindo, assim, manter a segurança e a privacidade dos professores e alunos registados no eTwinning). Os Grupos são encerrados pelo Serviço Central de Apoio após três meses de inactividade.

Após a aprovação do Grupo é aconselhável que o moderador coloque alguma informação antes de o abrir a todos. Caso contrário arrisca-se que os professores o visitem e não voltem mais por não encontrarem informação relevante.

Dentro destes Grupos existem alguns que são fechados, tendo sido criados pelo Serviço Central de Apoio para reunirem grupos de pessoas como os membros do Serviços Nacionais de Apoio ou membros de equipas mais restritas.

O espaço do grupo tem ferramentas básicas para ajudarem à dinâmica do mesmo.

-Diário do grupo; Páginas; Materiais; Fóruns; Em directo; Uma caixa de correio; 


\section{Grupos de Destaque eTwinning}

Os Grupos de Destaque eTwinning são espaços colaborativos com diversas temáticas que juntam à data deste artigo 38117 professores. Existem 12 grupos diferentes, sendo o espaço Creative Classroom o que congrega o maior número de membros $(7$ 050). Após o piloto com os 3 Grupos iniciais, criaram-se Grupos de Destaque que nasceram em Abril de 2014 e foram renovados em Setembro de 2015.

Os 12 Grupos de Destaque são da responsabilidade do CSS e são moderados por peritos ou professores convidados que receberam formação de moderadores. Alguns Grupos de destaque começaram por ser criados por professores eTwinners, e devido ao número de membros e da sua dinâmica passaram a ser apoiados e destacados pelo CSS.

\section{Grupos eTwinning criados por professores para professores}

A segunda tipologia de Grupos aparece na continuidade do espaço Salas dos Professores (Teachers Rooms) da plataforma anterior. Qualquer professor registado pode propor a criação de um grupo. A candidatura é avaliada pelo CSS. Pretende-se que sejam espaços agregadores e com espírito colaborativo e onde se fomente a partilha de práticas de forma a que os membros aprendam de e com os seus pares.

Os Grupos criados pelos professores surgem em Setembro de 2015. Desta data até Janeiro de 2016, 498 Grupos são abertos pelos professores eTwinners. No total 12352 membros aderem aos Grupos nos primeiros meses. No final de 2016, 1028 Grupos foram abertos e 24445 professores tornaram-se membros.

Em Setembro de 2016, ao fim de um ano do início desta nova fase, Portugal ocupava o sétimo lugar entre os 39 países com Grupos criados. Até à data deste artigo Portugal registou 3407 Grupos criados por professores, mantendo o sétimo lugar, com mais grupos criados, mas agora entre os 42 países que fazem parte do eTwinning.

\section{Grupos eTwinning - Percepções dos Professores}

Realizámos um questionário que foi proposto aos professores em vários grupos de Facebook que juntam eTwinners de todos os países parceiros. Recebemos 98 respostas de professores de 23 países diferentes. O questionário era composto por 11 perguntas sobre a utilização que os professores fazem do espaço da plataforma dedicada aos Grupos eTwinning. As perguntas foram concebidas para recolher informações sobre os membros dos Grupos enquanto participantes e enquanto criadores e moderadores dos Grupos.

\section{A Participação nos Grupos eTwinning - Percepções dos professores}

"The affective and social dimension of the learning process can be exploited to allow the learner to not only enjoy learning, but acquire skills that empower him to actively engage in the development of his personal skills and competences". (Redecker, 2009, p.60)
Pretendemos conhecer as percepções dos professores enquanto participantes nos Grupos eTwinning, tanto nos Grupos de Destaque como nos Grupos criados pelos professores. As perguntas realizadas não se centravam em qualquer das tipologias dos grupos, tendo sido colocadas de forma geral.

O questionário tinha 7 perguntas sobre a participação dos professores nos Grupos eTwinning.

1.Are you a member in any Featured Group? Resposta de sim ou não. No caso de resposta afirmativa deveriam escolher qual/quais do/s 12 Grupo/s de destaque faziam parte.

2.Do you participate in any eTwinning Group created by an eTwinner? Resposta de sim ou não.

3.If you answered yes in the previous question, write the name of the group/s. Pergunta de resposta aberta.

4.How often do you visit the group/s? Pergunta com resposta de escolha múltipla.

5.What do you enjoy more in an eTwinning group? Pergunta de resposta aberta.

6.What do you enjoy less in an eTwinning group? Pergunta de resposta aberta.

7.What do you look for in an eTwinning group? Please, share your impressions on using this tool. What are your impressions about the networking, collaboration, sharing that may have occurred during your participation? Or write any other comment on your experience on participating on groups. Pergunta de resposta aberta.

Dos 98 respondentes 94 participam nos Grupos de Destaque, sendo o Grupo Creative Classroom que tem um maior número de escolha dos respondentes $80,9 \%$ (tem 7050 membros, o Grupo de Destaque eTwinning com mais membros, $38,3 \%$ pertencem eTwinning for School Leadership - 38,3\% (937 membros), Game Based Classroom - 29,8\% (4 213 membros) e Virgilio an introduction to eTwinning - 27,7\% (6 062 membros). Os professores podem fazer parte de vários grupos ao mesmo tempo, sem limite. Em relação aos grupos criados por eTwinners a variedade de respostas é grande tendo em conta que na altura deste artigo existiam 1 028 Grupos com um total de 24445 membros. Algumas das respostas dadas demonstram que os professores não conhecem a diferença entre os grupos de Destaque e os Grupos criados por qualquer eTwinner.

Uma das recorrentes queixas dos professores é a falta de tempo. Perguntámos no questionário com que frequência visitavam os Grupos dos quais são membros. As opções eram as seguintes: "mais do que 1 vez por mês"; "menos do que 1 vez por mês"; "mais do que 1 vez por semana" "menos do que 1 vez por semana"; "Outros". As respostas apontam para um número baixo de visitas aos Grupos. A maioria dos respondentes seleccionaram a respostas "menos do que 1 vez por mês" (30.9\% das respostas) e por outro lado "mais do que 1 vez por semana" (25.8\% das respostas).

As restantes perguntas eram de resposta aberta e tinham como objectivo conhecer as opiniões dos professores sobre a utilização do Grupo.

Enquanto participantes os professores gostam dos Grupos devido aos momentos de partilha e de trabalho 
colaborativo que proporcionam. São reconhecidos como espaços onde se trocam experiência, saberes e práticas no espírito comunitário. A maioria dos respondentes refere os momentos vividos nos grupos como inspiradores que os ajudam a querer saber mais. "There I always learn new things." (AL2)

De acordo com as respostas recebidas os professores procuram nos Grupos eTwinning inspiração, ideias novas, oportunidades para trabalhar com os seus pares, informação/conhecimento, conselhos e apoio.

Destacamos os seguintes comentários que ilustram o que os membros dos Grupos procuram.

-"Sharing REAL experiences, good practices. There is ALWAYS a person to give useful advice." (BG1)

- "Aprender o mais possível e tentar com isso melhorar a minha forma de ensinar, tentar inovar e ser mais criativa para "sair da rotina" e principalmente, incentivar os meus alunos para a aprendizagem para que possam efetivamente adquirir as competências necessárias para o século XXI."(PT6)

-"eTwinning Groups are an excellent way for learning from our peers. I expect to find mainly inspiration and advice. I also hope that my contributions have been helpful to someone else. Groups are (can be) a place where we share what we are doing inside our classroom, knowing that the teachers on the other side will understand us because we "talk the same language". So, in the end, I may say that groups are also a place to meet friends!"“(PT7)

Os professores apreciam a disponibilidade do outro em partilhar. "I like the collaborative element \& how others are willing to share ideas \& practice." (Uk1) "As a beginner, I appreciated the disinterested mutual support of the members. They gave me reasons and strength to pass by obstacles and move on with the project.." (PT18) Além disso acreditam que todas as dinâmicas e oportunidades que esta plataforma fornece os ajuda a crescer enquanto profissionais e pessoas, "I am confident that - in multiple and various ways - each little bit that I add myself and/or enjoy as being added by others to the groups I am part of, has helped and shaped my identity as the eTwinner I am today." (RO9).

\section{A criação dos Grupos eTwinning - Percepções dos professores}

"Learning in the digital era is fundamentally collaborative in nature; social networks arise around common (learning) interests and aims and facilitate the learning process by providing social and cognitive guidance and support" (Redecker, 2009, p.9).

Pretendemos conhecer as percepções dos professores enquanto criadores de Grupos eTwinning. O questionário dividia-se em dois grupos de perguntas. Apenas os professores que respondessem à pergunta 8 , "Have you created a group?" (Resposta de sim ou não) avançavam para outro conjunto de 4 perguntas específica para professores que tivessem criado Grupos eTwinning. Ao responderem não, passavam para a última secção.

Grupo de perguntas realizadas aos professores que tenham criado um Grupo.
1. How many groups have you created? Resposta com hipóteses e campo aberto de outro.

2. Was it easy to create the group? Resposta de sim ou não.

3. As a moderator do you use all the tools available? Resposta de sim ou não. Opção de acrescentar comentários.

4. Please, share your impressions on moderating a group. What are your impressions about the networking, collaboration, sharing that may occur ? Or write any other comment on your experience on moderating a group. Pergunta de resposta aberta.

Todas as perguntas eram de resposta obrigatória. Dos 98 respondentes ao questionário 25 responderam que criaram Grupos eTwinning (25,5\%).

A maioria dos respondentes, 19 professores $(76 \%)$, criou 1 grupo apenas e os restantes 6 professores (24\%) criaram 2.

Em relação à facilidade ou não de criação grupos 23 respondentes $(92 \%)$ consideram que é fácil o processo de criação. 56\% dos respondentes afirma que não utilizam todas as ferramentas disponíveis nos grupos. Apesar de existir a oportunidade de fazer comentários a esta pergunta, estes não se referem na maioria à pergunta feita. Nas respostas pelo menos dois respondentes demonstram a necessidade saber mais sobre as ferramentas disponíveis. No que concerne às ferramentas um respondente refere-se às mesmas noutro espaço para comentários: "It's quite easy to handle the tools but really demanding to find interesting topics and ways to motivate members to participate and be willing to share their ideas" (EL6)

Os professores foram convidados a partilhar comentários no geral sobre a moderação dos grupos. As respostas foram variadas e apresentam testemunhos sobre as vantagens pessoais de moderar um grupo, assim como, as dificuldades que enquanto moderadores os professores encontram nos grupos.

Os professores moderadores consideram que criar e manter a actividade num Grupo eTwinning é desafiante por várias razões. A comunicação entre os membros é difícil de promover e manter. "It was challenging because I had to find interesting ways to keep the members connected to the groups." (RO2) Uma das razões que apresentam é a falta de um sistema de notificações imediato que permita que os membros do grupo saibam que estão a ser contactados ou que ocorreu alguma interacção.

Uma das vantagens apontadas pelos professores enquanto participantes na utilização dos Grupos é para os moderadores algo que pode ser frustrante, a partilha. Os moderadores afirmam que a maioria dos membros dos Grupos não partilham informação nem interagem limitando-se a procurarem informação partilhada por alguns membros mais activos. Muitos moderadores consideram que os grupos sofrem com a sua falta de tempo para gerir o espaço, pensar em actividades, promover a discussão. Por um lado consideram que ser moderador é uma obrigação mas também uma motivação. "It's too much work to keep the group active and members motivated. Communication among members is difficult too. Still, I believe that it's 
important to have like-minded or sharing same interests people together and offer the opportunity to share." (EL2). Na pergunta (4) de resposta aberta na qual solicitávamos que partilhassem as suas percepções sobre a moderação de um grupo, os moderadores dão as mesmas respostas que deram nas perguntas sobre a sua experiência enquanto participantes dos Grupos. Enquanto moderadores procuram inspiração, ideias novas, trabalhar com os seus pares, conhecimentos, conselhos e apoio. "I created my own group, because I would like to develop my ICT skills and experience." (PL4) Ao promoverem um espaço de partilha e de colaboração os moderadores dos Grupos consideram que estão a aprender ao mesmo tempo que promovem e facilitam a aprendizagem de outros. "Learn more than you think." (AL2)

O espaço dos Grupos tem uma caixa de correio, contudo só quando de se entra na plataforma que gere os grupos é que o professor sabe que alguém o tentou contactar. o mesmo acontece com as interacções que acontecem a nível dos fóruns. Apesar de existir um espaço de destaque sobre as últimas contribuições dos fóruns estas só são acessíveis se os membros entrarem na plataforma dos Grupos. Apesar destes constrangimentos as respostas ao questionário apontam para preferencialmente para pontos positivos (todas as conclusões serão partilhadas com o Serviço Central de Apoio do projecto).

\section{Conclusão}

"They help me to broaden horizons and be in contact with a large community of people with common interests." (EL8)

Os professores respondentes valorizam diferentes características da ferramenta, nomeadamente o facto de esta lhes dar a oportunidade de aprenderem com os seus pares, se inspirarem com as partilhas de ideias e experiências, os contributos, a disponibilidade dos outros em ajudar. Consideram que o ponto mais forte é a oportunidade que lhes dá para conhecer novas pessoas de diferentes países que partilham os mesmos gostos, dúvidas e medos, a possibilidade de partilhar práticas, de colaborar e aprender uns com os outros de forma interactiva. Os professores afirmam que se tornaram mais confiantes nas suas práticas e mais motivados.

A ferramenta Grupos eTwinning responde a algumas das necessidades dos professores enquanto aprendentes preocupados com o seu desenvolvimento pessoal e profissional. Os professores valorizam muito estes momentos voluntários de aprendizagem, mudando a percepção de que o conhecimento está confinado à escola, aos centros de formação e aos seus formadores. $\mathrm{O}$ desenvolvimento tecnológico fez com que aprendizagem possa acontecer em qualquer lugar a qualquer hora. Todos podem ser peritos e podem aprender uns com os outros. Como em qualquer outra comunidade nem todos contribuem ao mesmo nível, contudo o número de momentos de partilha $\mathrm{e}$ colaboração entre os pares continua a crescer.

Tendo em conta o aumento de interesse por esta ferramenta será importante tirar conclusões no futuro sobre o verdadeiro impacto que estes momentos têm no desenvolvimento pessoal e profissional dos professores

\section{Referências}

Crawley, C., Gilleran, A., Scimeca, S., Vuorikari, R. \& Wastiau,P. (2009). Beyond School Projects - A report on eTwinning 2008-2009. Brussels: Central Support Service of eTwinning European Schoolnet.

Crawley, C., Gerhard, P., Gilleran, A.,Joyce, A. (2010). eTwinning 2.0 - Building the community for schools in Europe. Brussels: Central Support Service of eTwinning European Schoolnet.

European Schoolnet (2013). Survey of Schools: ICT in Education. Benchmarking access, use and attitudes to technology in Europe's schools. Executive Summary. Acedido em 21 de Fevereiro 2017, em

http://www.eun.org/c/document_library/get_file?uuid=9 be81a75-c868-4558-a777-

862 ecc8162a4\&groupId $=43887$

European Schoolnet (200). First year of eTwinning in Europe. Acedido em 3 de Março 2017 https://files.eTwinning.net/shared/data/eTwinning/bro chures/etw_leaflet_en.pdf

Kearney, C. \& Gras-Velázquez, À. (2015). eTwinning Ten Years On: Impact on teachers' practice, skills, and professional development opportunities, as reported by eTwinners. Brussels: Central Support Service of eTwinning European Schoolnet.

Redecker, C. (2009). Review of Learning 2.0 Practices: Study on the Impact of Web 2.0 Innovations on Education and Training in Europe. Retirado de Institute for Prospective Technological Studies, JRC, European Commission, acedido em 29 de Abril 2017: http://ipts.jrc.ec.europa.eu/publications/pub.cfm?id=205 9. 